\title{
Inverse population synthesis using a dynamical basis
}

\author{
Juan Mateu ${ }^{1}$, Gladis Magris ${ }^{2}$ and Gustavo Bruzual ${ }^{2}$ \\ ${ }^{1}$ Universidad de Carabobo, Venezuela \\ email: mateu@uc.edu.ve \\ ${ }^{2}$ Centro de investigaciones de Astronomía, CIDA, Venezuela
}

\begin{abstract}
We introduce a new inverse population synthesis algorithm (DINBAS3D) which aims to recover the star formation and metallicity histories from galactic spectra. We investigate the use of a dynamical basis of three simple stellar population spectra that is specific for each galaxy. Our goal is to recover a robust star formation history that minimizes degeneracy effects which are very common in high resolution histories methods. In this work, we detail the method and present our findings when we apply DINBAS3D to synthetic spectra with known parameters, we compare our results with similar methods and find good agreement between them.
\end{abstract}

Keywords. Galaxy: evolution

\section{Introduction}

A galaxy is composed mainly of stars, interstellar gas and dust, however, the stars contribute the most to the total luminosity of a galaxy. The Method of Stellar Population Synthesis consists in representing the spectrum of a galaxy as a linear combination of their stellar components spectra, leaving aside minor contributions due to presence of gas and dust.

In recent years, several techniques have been developed to recover the star formation history and chemical evolution from observed galaxy spectrum, this techniques are known as Inverse Population Synthesis.

Most of the methods of Inverse Population Synthesis (Reichardt et al. 2001, CidFernandes et al. 2005, Tojeiro et al. 2007) use a linear combination of a fixed set (basis) of simple stellar population spectra (Bruzual and Charlot 2003) to fit the observed spectrum and a maximum likelihood parameter (coefficients) estimation to find the best set of parameters that corresponds to that fit. We developed a new algorithm where the basis changes to adapt and optimize the appropriate fitting of the spectra, this method is called dynamical basis.

\section{The Dynamical Basis}

The simplest way to simulate a composed stellar population is using a Simple Stellar Population with some particular mass and age, that would be a 1D Dynamical Basis, it works pretty good for early type galaxies, but it does not work very good for composed stellar populations like spirals and irregulars. The idea of a dynamical basis is to add other elements to this basis that can be of any age, the algorithm will search for the best combination of age and mass of each component that best fits the observed spectrum.

In our algorithm DINBAS3D, we use $N=3$ elements in the basis. The process begins by minimizing a merit function like $\chi^{2}$ that measures the agreement between observed 
and modeled spectra:

$$
\chi^{2}\left(t_{1}, t_{2}, \ldots, t_{N}\right)=\sum_{i=1}^{M} \frac{\left(f_{o}\left(\lambda_{i}\right)-\sum_{j=1}^{N} a_{j} f\left(\lambda_{i}, t_{j}\right)\right)^{2}}{\sigma_{i}^{2}}
$$

where $f_{o}\left(\lambda_{i}\right.$ is the observed spectrum, $f\left(\lambda_{i}, t_{j}\right)$ is the Simple Stellar Population spectrum of age $t_{j}, a_{j}$ is the mass formed at that age and $\sigma_{i}$ is the standard deviation of the noise in the observed spectrum. We can also consider metallicity variations by adding other set of Simple Stellar Populations. Coefficients $a_{j}$ are calculated by solving the following set of linear equations:

$$
\sum_{j=1}^{N} a_{j} \sum_{i=1}^{M} \frac{f\left(\lambda_{i}, t_{k}\right) f\left(\lambda_{i}, t_{j}\right)}{\sigma_{i}^{2}}=\sum_{i=1}^{M} \frac{f_{o}\left(\lambda_{i}\right) f\left(\lambda_{i}, t_{k}\right)}{\sigma_{i}^{2}}
$$

with the restriction of $a_{j} \geqslant 0$.

\section{Results}

We applied the dynamical basis method and the fixed basis method to a synthetic 5 burst composed stellar population. The global behavior of this star formation history is recovered with both methods. In the Dynamical Basis method the fitting improves as the number of elements in the basis is increased. We obtain the same excellent fit with a 3 element basis (3D) as in a 15 element fixed basis.

Conventional methods that use fixed basis works under the premise that the set of Simple Stellar Population spectra that they use as a basis is actually capable of generate a spectrum of any composed stellar population. In particular, the spectra that form the "base" should be able to generate the simplest possible case, i.e. the spectrum of a simple stellar population. However,we made a comparison between a method of fixed base (with the same basis of 15 elements as in Cid-Fernandes et al. 2005) and our dynamic basis algorithm DINBAS3D applied to a set of synthetic spectra of simple stellar populations of different ages, we have also calculated $\chi^{2}$ values corresponding to the minimum possible difference between the spectrum with and without noise. We observe in this experiment that for the fixed basis method, the adjustment is good for most ages, but some of them, the fitting becomes quite difficult, which means that our initial premise is not totally true, in contrast, with DINBAS3D we obtained perfect fitting for all ages.

\section{Conclusions}

We developed a new method for Inverse Population Synthesis which linearly combines a non-static Simple Stellar Population spectra to generate any composed population spectra. We also compare the method with traditional methods that use a fixed basis and obtained better or equally good fitting and simpler Star Formation Histories.

\section{References}

Bruzual, G. \& Charlot, S. 2003, MNRAS, 344, 1000

Cid Fernandes, R., Mateus, A., Sodré, L., Stasinska, G., \& Gomes, J. M. 2005, MNRAS, 358, 363

Reichardt, C., Jimenez, R., \& Heavens, A. F. 2001, MNRAS, 327, 849

Tojeiro, R., Heavens, A. F., Jimenez, R., \& Panter, B. 2007, MNRAS, 381, 1252 